\title{
Phase II Study of S-1 Plus Either Irinotecan or Docetaxel for Non-small Cell Lung Cancer Patients Treated with More Than Three Lines of Treatment
}

\section{Dal Yong Kim, MD \\ Dae Ho Lee, MD \\ Sun-Joo Jang, MD \\ Sang-We Kim, MD \\ Cheolwon Suh, MD \\ Jung Shin Lee, MD}

Department of Oncology, Asan Medical Center, University of Ulsan College of Medicine, Seoul, Korea
Correspondence: Dae Ho Lee, MD, PhD Department of Oncology, Asan Medical Center, University of Ulsan College of Medicine, Pungnap 2-dong, Songpa-gu,

Seoul 138-736, Korea

Tel: 82-2-3010-3214

Fax: 82-2-3010-6961

E-mail: leedaeho@amc.seoul.kr

Received July 12, 2011

Accepted August 6, 2011

\section{Purpose}

This study was designed to evaluate the efficacy of a combination treatment of S-1 plus either irinotecan or docetaxel for advanced/metastatic non-small cell lung cancer (NSCLC) patients who have already failed 3 or more lines of treatment.

\section{Materials and Methods}

This was a prospective single center phase II study. The eligible patients received S-1 $40 \mathrm{mg} / \mathrm{m}^{2}$ twice a day orally on days 1 though 14 combined with irinotecan $150 \mathrm{mg} / \mathrm{m}^{2}$ on D1 only or docetaxel $35 \mathrm{mg} / \mathrm{m}^{2}$ on D1 and D8. The treatment was repeated every 3 weeks until disease progression, unacceptable toxicity, or patient refusal. The choice between the two regimens was made at the discretion of the treating physician.

\section{Results}

A total of 14 patients participated in the study. There were 3 patients with squamous cell carcinoma, 9 with adenocarcinoma, and 2 with NSCLC, NOS. Eight of the patients were male. There were 8 patients with an Eastern Cooperative Oncology Group (ECOG) of 1, and 6 patients with an ECOG of 2. All the patients had already been treated with platinum-based chemotherapy and epidermal growth factor receptor tyrosine kinase inhibitor therapy. Out of the 14 patients, 10 received irinotecan and S-1 and the other 4 received docetaxel and S-1. Twelve patients had also received pemetrexed. Disappointingly, there were no response from 2 patients with a stable disease, and therefore, as per the protocol, we stopped the study early. With a median follow-up time of 49 months, the median survival time was 5.6 months ( $95 \%$ confidence interval, 4.3 to 6.9 months).

\section{Conclusion}

S-1 containing doublets did not show activity in this population as a salvage treatment and further investigation cannot be recommended.

\section{Introduction}

Patients with advanced or metastatic non-small cell lung cancer (NSCLC) benefit from a platinum-containing doublet [1]. In addition, recently developed monoclonal antibodies, such as cetuximab and bevacizumab, can be more beneficial in combination with chemotherapy for a selected subset of these patients [2,3]. As a second-line or third-line therapy, single agent docetaxel, pemetrexed and oral epidermal growth factor receptor tyrosine kinase inhibitor (EGFR TKI) improved the survival out-

\section{Key words}

Non-small-cell lung carcinoma, Salvage therapy, S-1, Irinotecan, Docetaxel 
ypyridine enhances the serum concentration of 5-FU by the competitive inhibition of dihydropyrimidine dyhydrogenase, which is an enzyme responsible for 5-FU catabolism. Potassium oxonate is also a reversible competitive inhibitor of oratate phosphoribosyl transferase, a phosphoenzyme for 5-FU. The diarrhea induced by 5-FU administration is thought to be attributable to the phosphorylation of 5-FU by enzyme in the gastrointestinal tissue, and after the oral administration of potassium oxonate, the concentration of potassium in the gastrointestinal tissue is high enough to inhibit the enzyme while the concentration of potassium in the blood and tumor is reported to be either slight or nil [9]. Because of these mechanisms, oral S-1 administration generates a higher concentration of 5-FU than a protracted intravenous infusion of 5-FU given in a dose equimolar to the tegafur in S-1, whereas the incidence of adverse events concerning the gastrointestinal tract does not increase. In a phase III trial of S-1 plus carboplatin in the first-line setting of NSCLC, S-1 plus carboplatin was not inferior to paclitaxel plus carboplatin in terms of the survival outcome. S-1 showed activity in a second-line setting as well [10]. Of more interest, single agent $\mathrm{S}-1$ showed modest activity as a third-line or further-line treatment $[11,12]$. On the other hand, capecitabine, which is another oral 5FU agent, showed clinically meaningful results in NSCLC when combined with irinotecan and docetaxel in both first-line and second-line settings [13-16].

Based on those findings, we conducted a phase II trial of S-1 containing doublets, that is, S-1 plus either irinotecan or docetaxel, for patients who had already received 3 lines of therapy or more, including at least a platinum doublet and an EGFR TKI.

\section{Materials and Methods}

\section{Eligibility}

The eligible patients were diagnosed with histologically or cytologically confirmed metastatic NSCLC. They had already received at least 3 lines of treatment, including a platinum doublet and EGFR TKI therapy. The other inclusion criteria were an age of 18 years and older, a Eastern Cooperative Oncology Group (ECOG) performance status of 0 to 2, measurable disease by the Response Evaluation Criteria in Solid Tumors (RECIST) v1.0 and adequate bone marrow, renal, and hepatic function. Patients with newly-diagnosed central nervous system metastases or any unresolved chronic toxicity greater than National Cancer Institute Common Terminology Criteria for Adverse Events (NCI CTCAE) grade (G) 2 from previous therapy were ineligible. All the patients provided written informed consent before starting the study. The study was approved by the Asan Medical Center Institutional Review Board (No.2006-0413) and the study was performed in accordance with the Declaration of Helsinki and the International Conference on Harmonisation/Good Clinical Practice.

\section{Treatment}

The treatment consisted of oral S-1 $40 \mathrm{mg} / \mathrm{m}^{2}$ twice a day from D1 through D14 in combination of either irinotecan $150 \mathrm{mg} / \mathrm{m}^{2}$ on D1 only or docetaxel $35 \mathrm{mg} / \mathrm{m}^{2}$ on D1 and D8, and this was repeated every 3 weeks. The actual dose of S- 1 was selected as follows: $40 \mathrm{mg}$ twice a day was given for a body surface area (BSA) $<1.25 \mathrm{~m}^{2}, 50 \mathrm{mg}$ twice a day was given for a BSA of $1.25 \mathrm{~m}^{2}$ but $<1.5 \mathrm{~m}^{2}$ and $60 \mathrm{mg}$ twice a day was given for a BSA $\geq 1.5 \mathrm{~m}^{2}$. Prophylactic antiemetics, a 5-HT3 receptor antagonist and $8 \mathrm{mg}$ of dexamethasone were given 30 minutes before the administration of irinotecan or docetaxel. However, further prophylactic dexamethasone after docetaxel was not routinely administered in order to reduce the risk of a hypersensitivity reaction. There was no concomitant medication for S-1 either. The choice between the two treatments was made at the treating physician's discretion.

Before receiving treatment, each patient underwent a complete physical examination, including laboratory tests, as well as a medical history evaluation, including documentation of concomitant medications and determining the performance status. Toxicities were graded using the CTCAE v3.0. In addition, all the patients received chest radiographs and a chest computed tomography scan that included the upper abdomen. Magnetic resonance imaging of the brain and radionuclide bone scans were done if needed.

Dose reductions and/or administration delays were planned in case of severe hematological and/or non-hematological toxicities while on the study's treatment. Dose adjustments were made according to the system showing the greatest degree of toxicity. Toxicities were graded using the CTCAE v3.0. The doses of irinotecan or docetaxel and S-1 were adjusted according to the degree of the hematologic and non-hematologic toxicity. The dose was reduced by one dose level of irinotecan $25 \mathrm{mg} / \mathrm{m}^{2}$ or docetaxel $5 \mathrm{mg} / \mathrm{m}^{2}$ and S-1 $20 \mathrm{mg}$ per day for the patients whose BSA was $\geq 1.25 \mathrm{mg}$ and who had evidence of G4 hematologic toxicity or G3 or more non-hematologic toxicity during any cycle of administration. If recovery from such toxicities was confirmed at a reduced dose, then administration at the reduced dose was continued. If a patient with a BSA $<1.25 \mathrm{~m}^{2}$ or who was taking $40 \mathrm{mg}$ twice a day experienced the abovementioned toxicities, then no further treatment with S-1 was done. If a rest period of $>4$ weeks was required, then the patient was withdrawn from the study. If reduction of more than two dose levels was required, then the patient was withdrawn from the study.

\section{Response assessment}

The objective tumor response was assessed according to RECIST v1.0. A complete response (CR) was defined as the disappearance of all lesions. A partial response (PR) was defined as at least a 30\% decrease in the sum of the longest target lesion diameter, taking as a reference the longest baseline diameter and/or the persistence of one or more non-target lesions. PD was defined as at least a $20 \%$ increase in the sum of the longest diameter, taking as a reference the smallest sum of the longest diameter recorded after treatment or the appearance of one or more new lesions, or the un- 
equivocal progression of existing non-target lesions. Stable disease (SD) was defined as the absence of significant shrinkage or enlargement that qualified as CR, PR or PD, taking as a reference the smallest sum of the longest diameter recorded after treatment. The best tumor response of $\mathrm{CR}$, PR or SD should be confirmed and sustained for at least 6 weeks or longer. The response was assessed every 2 cycles. Progression-free survival (PFS) was defined as the interval between the date treatment started and the date of documented disease progression or death from any cause. Overall survival was defined as the interval between the date treatment started and the date of death from any cause. Patients lost to follow-up were censored at the last date of contact.

\section{Statistical considerations}

This study was an open label, non-randomized, single-institution, single-arm phase II study. The primary endpoint was the objective response rate. A Simon mini-max two-stage design was chosen to determine the total number of patients required for the phase II study. We set a response rate of $30 \%$ as the target activity level and we chose $10 \%$ as the lowest overall response rate of interest. The study was designed to have $80 \%$ power to accept the hypothesis and 5\% significance to reject the hypothesis. For a total of 25 subjects, 15 were to be accrued during the first stage and 10 were to be accrued during the second stage. If 1 or fewer responses were observed during the first stage, then the study would stop early. If 5 or fewer responses were observed by the end of the study, then no further investigation of the drug would be warranted. Allowing a loss to followup rate of $10 \%$, a total of 28 patients were required. The data was updated as of June 30, 2011.

\section{Results}

\section{Patient characteristics}

Between March 2007 and July 2009, 14 patients participated in the study. Among them, 10 patients received irinotecan plus S-1 and the other 4 patients received docetaxel plus S- 1 . The characteristics of the patients are listed in Table 1. As per protocol, all the patients received platinumbased chemotherapy and EGFR TKI therapy.

\section{Tumor responses in patients and survival}

Out of the 14 patients there was no response, and only 2 showed SDs. Therefore, we had to stop the study early as per protocol. With a median follow-up time of 49 months, the median survival time was 5.6 months ( $95 \%$ confidence interval [CI], 4.3 to 6.9 months) (Fig. 1). The median PFS was 1.4 months ( $95 \%$ CI, 0 to 3.0 months).
Table 1. Patient characteristics

$\begin{array}{lcc}\text { Characteristic } & \text { No. }(\mathbf{n = 1 4}) & \% \\ \text { Median age (range, yr) } & 59(45-72) & \\ \text { Gender } & 8 & 57.1 \\ \quad \text { Male } & 6 & 42.9 \\ \quad \text { Female } & & \\ \text { ECOG performance status } & 8 & 57.1 \\ 1 & 6 & 42.9 \\ 2 & & \\ \text { Pathology } & 3 & 21.4 \\ \text { Squamous cell carcinoma } & 9 & 64.3 \\ \text { Adenocarcinoma } & 2 & 14.3 \\ \quad \text { Non-small cell lung cancer, NOS } & & \\ \text { No. of prior chemotherapies including } & & 71.4 \\ \text { EGFR TKI therapy } & 10 & 21.4 \\ 3 & 3 & 7.1 \\ 4 & 1 & \\ 5 & \end{array}$

ECOG, Eastern Cooperative Oncology Group; NOS, not ot-herwise specified; EGFR TKI, epidermal growth factor receptor tyrosine kinase inhibitor.

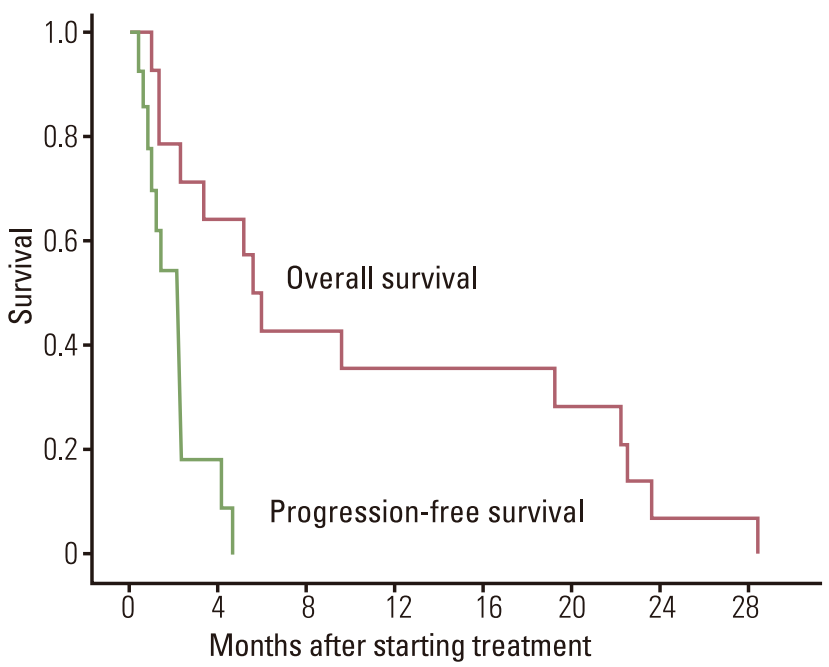

Fig. 1. Survival outcomes.

\section{Toxicity}

There were 3 patients with $\mathrm{G} 2$ hematologic toxicity, 2 patients had G1 anemia, 1 patient had G3 neutropenia, and 1 patient had G2 neutropenia. One patient experienced G3 diarrhea and 1 patient experienced G3 nausea/vomiting. All the patients receiving S-1 and docetaxel manifested alopecia. The other toxicities were G1/2 toxicities such as fatigue, mucositis, etc., which were tolerable and manageable (Table 2). 
Table 2. Toxicity profile

\begin{tabular}{|c|c|c|c|c|c|c|c|c|}
\hline \multirow{2}{*}{ Adverse event } & \multicolumn{4}{|c|}{ S-1 plus irinotecan $(n=10)$} & \multicolumn{4}{|c|}{ S-1 plus docetaxel $(n=4)$} \\
\hline & G1 & G2 & G3 & G4 & G1 & G2 & G3 & G4 \\
\hline \multicolumn{9}{|l|}{ Hematologic toxicity } \\
\hline Neutropenia & $0(0)$ & $1(10)$ & $1(10)$ & $0(0)$ & $0(0)$ & $0(0)$ & $0(0)$ & $0(0)$ \\
\hline Anemia & $2(20)$ & $3(30)$ & $0(0)$ & $0(0)$ & $1(25)$ & $0(0)$ & $0(0)$ & $0(0)$ \\
\hline Thrombocytopenia & $0(0)$ & $0(0)$ & $0(0)$ & $0(0)$ & $0(0)$ & $0(0)$ & $0(0)$ & $0(0)$ \\
\hline \multicolumn{9}{|c|}{ Non-hematologic toxicity } \\
\hline Alopecia & $0(0)$ & $0(0)$ & $0(0)$ & $0(0)$ & $3(75)$ & $1(25)$ & $0(0)$ & $0(0)$ \\
\hline Diarrhea & $0(0)$ & $0(0)$ & $1(10)$ & $0(0)$ & $0(0)$ & $0(0)$ & $0(0)$ & $0(0)$ \\
\hline Fatigue & $0(0)$ & $3(3)$ & $0(0)$ & $0(0)$ & $0(0)$ & $1(25)$ & $0(0)$ & $0(0)$ \\
\hline Nausea/Vomiting & $0(0)$ & $0(0)$ & $1(10)$ & $0(0)$ & $0(0)$ & $0(0)$ & $0(0)$ & $0(0)$ \\
\hline Elevated AST/ALT & $0(0)$ & $0(0)$ & $0(00)$ & $0(0)$ & $0(0)$ & $0(0)$ & $0(0)$ & $0(0)$ \\
\hline Elevated bilirubin & $1(10)$ & $0(0)$ & $1(10)$ & $0(0)$ & $0(0)$ & $0(0)$ & $0(0)$ & $0(0)$ \\
\hline
\end{tabular}

Values are presented as number (\%). AST, aspartate aminotransferase; ALT, alanine aminotransferase.

\section{Discussion}

Regrettably we did not observe the activity of a combination of S-1 and either irinotecan or docetaxel as a fourth-line or further-line therapy in heavily-treated NSCLC patients. As per the protocol, we had to stop the study early, which meant that we cannot recommend these regimens for further investigation. In a retrospective study of a single S-1 agent in a clinical setting, the authors reported that $5.7 \%$ of the enrolled patients showed a PR and $34.2 \%$ had SD. Another retrospective study of pemetrexed or docetaxel as third or fourth-line therapy also showed an objective response rate of $15.4 \%$ [17]. But those studies might have inherent limitations due to their retrospective nature. Our prospective study did not confirm this kind of efficacy. The advances of treatment for NSCLC during the last decade cause physicians to frequently see patients who have PD after multiple chemotherapies, but they still have a good performance status. As a result, they still have a chance to receive further cytotoxic chemotherapy, when considering their performance status and the unused, available chemotherapeutic agents. Actually, in our study, all the patients had already received a platinum doublet and either gefitinib or erlotinib or both, and most of them had also received either pemetrexed or docetaxel. For such patients, many investigators, including us, have conducted clinical trials of single or combination chemotherapy of cytotoxic agents that have already showed their activity as first-line and second-line treatments. They have also reported a few responses in phase II trials, but their results hardly translated into success in the phase III trials. Like many other studies, a rather unselected population participated in our study and so a lower efficacy of the treatment could be expected, especially for heavily-treated patients. We determined the response rate as the primary endpoint, and we would have chosen the PFS rate at some point instead of the response rate as the primary endpoint. However, unlike targeted agents, which are sometimes regarded as cytostatic drugs, most chemotherapeutic agents are cytotoxic drugs, so the response rate might be better as the primary endpoint for cytotoxic agents than the progression-free rate [18].

On the other hand, we need to change the strategy of clinical trials in this type of clinical setting. The most promising one might be to design clinical trials based on the patients' molecular characteristics, which are usually discovered in other studies. For example, since information on specific genotypes related to the pemetrexed-metabolizing genes, such as SLC19A1, might be related to the clinical outcome, we can consider conducting a clinical trial based on this genetic information [19]. Another strategy should focus on many biomarkers such as epidermal growth factor receptor (EGFR) and KRAS mutations, the copy number of cyclin D1, the expression of vascular endothelial growth factor, or molecular driver mutations such as the echinoderm microtubule-associated protein-like 4 anaplastic lymphoma kinase (EML4-ALK) fusion gene or $b$-raf mutation. We recommend that patients participate in clinical trials of newly-developed targeted agents for such targets [20,21].

\section{Conclusion}

Treatment with S-1 and either irinotecan or docetaxel did not yield efficacy in heavily-treated advanced NSCLC patients as fourth-line or further-line therapy, and so this treatment cannot be recommended.

\section{Conflicts of Interest}

Conflict of interest relevant to this article was not reported. 


\section{References}

1. Schiller JH, Harrington D, Belani CP, Langer C, Sandler A, Krook J, et al. Comparison of four chemotherapy regimens for advanced non-small-cell lung cancer. N Engl J Med. 2002; 346:92-8.

2. Pirker R, Pereira JR, Szczesna A, von Pawel J, Krzakowski M, Ramlau R, et al. Cetuximab plus chemotherapy in patients with advanced non-small-cell lung cancer (FLEX): an openlabel randomised phase III trial. Lancet. 2009;373:1525-31.

3. Sandler A, Gray R, Perry MC, Brahmer J, Schiller JH, Dowlati A, et al. Paclitaxel-carboplatin alone or with bevacizumab for non-small-cell lung cancer. N Engl J Med. 2006;355:254250 .

4. Shepherd FA, Dancey J, Ramlau R, Mattson K, Gralla R, O'Rourke M, et al. Prospective randomized trial of docetaxel versus best supportive care in patients with non-small-cell lung cancer previously treated with platinum-based chemotherapy. J Clin Oncol. 2000;18:2095103.

5. Hanna N, Shepherd FA, Fossella FV, Pereira JR, De Marinis F, von Pawel J, et al. Randomized phase III trial of pemetrexed versus docetaxel in patients with non-small-cell lung cancer previously treated with chemotherapy. J Clin Oncol. 2004;22:1589-97.

6. Shepherd FA, Rodrigues Pereira J, Ciuleanu T, Tan EH, Hirsh V, Thongprasert S, et al. Erlotinib in previously treated non-small-cell lung cancer. N Engl J Med. 2005;353:123-32.

7. Kim ES, Hirsh V, Mok T, Socinski MA, Gervais R, Wu YL, et al. Gefitinib versus docetaxel in previously treated non-small-cell lung cancer (INTEREST): a randomised phase III trial. Lancet. 2008:372:1809-18.

8. Shirasaka T, Shimamato Y, Ohshimo H, Yamaguchi M, Kato T, Yonekura K, et al. Development of a novel form of an oral 5 -fluorouracil derivative (S-1) directed to the potentiation of the tumor selective cytotoxicity of 5-fluorouracil by two biochemical modulators. Anticancer Drugs. 1996;7:548-57.

9. Shirasaka T, Shimamoto Y, Fukushima M. Inhibition by oxonic acid of gastrointestinal toxicity of 5-fluorouracil without loss of its antitumor activity in rats. Cancer Res. 1993;53:4004-9.

10. Okamoto I, Yoshioka H, Morita S, Ando M, Takeda K, Seto T, et al. Phase III trial comparing oral S-1 plus carboplatin with paclitaxel plus carboplatin in chemotherapy-naïve patients with advanced non-small-cell lung cancer: results of a west Japan oncology group study. J Clin Oncol. 2010;28:5240-6.

11. Govindan R, Morgensztern D, Kommor MD, Herbst RS, Schaefer P, Gandhi J, et al. Phase II trial of S-1 as second-line therapy in patients with advanced non-small cell lung cancer. J Thorac Oncol. 2011;6:790-5.

12. Ono A, Naito T, Murakami H, Takahashi T, Nakamura Y, Tsuya A, et al. Evaluation of S-1 as third- or further-line chemotherapy in advanced non-small-cell lung cancer. Int J Clin Oncol. 2010;15:161-5.

13. Han JY, Lee DH, Kim HY, Hong EK, Yoon SM, Chun JH, et al. A phase II study of weekly docetaxel plus capecitabine for patients with advanced nonsmall cell lung carcinoma. Cancer. 2003;98:1918-24.

14. Han JY, Lee DH, Kim HY, Kim EA, Lee JJ, Ju SY, et al. A Phase II study of weekly irinotecan and capecitabine in patients with previously treated non-small cell lung cancer. Clin Cancer Res. 2003;9(16 Pt 1):5909-14.

15. Han JY, Lee DH, Lee SY, Park CG, Kim HY, Lee HG, et al. Phase II study of weekly irinotecan plus capecitabine for chemotherapy-naïve patients with advanced nonsmall cell lung carcinoma. Cancer. 2005;104:2759-65.

16. Lee JJ, Han JY, Lee DH, Kim HY, Chun JH, Lee HG, et al. A phase II trial of docetaxel plus capecitabine in patients with previously treated non-small cell lung cancer. Jpn J Clin Oncol. 2006;36:761-7.

17. Chen YM, Shih JF, Fan WC, Wu CH, Chou KT, Tsai CM, et al. Third-line or fourth-line chemotherapy in non-small-cell lung cancer patients with relatively good performance status. J Chin Med Assoc. 2011;74:209-14.

18. Fox E, Curt GA, Balis FM. Clinical trial design for target-based therapy. Oncologist. 2002; 7:401-9.

19. Adjei AA, Salavaggione OE, Mandrekar SJ, Dy GK, Ziegler KL, Endo C, et al. Correlation between polymorphisms of the reduced folate carrier gene (SLC19A1) and survival after pemetrexed-based therapy in non-small cell lung cancer: a North Central Cancer Treatment Group-based exploratory study. J Thorac Oncol. 2010;5:1346-53.

20. Kim ES, Herbst RS, Wistuba II, Lee JJ, Blumenschein GR, Tsao A, et al. The BATTLE tiral: personalizing therapy for lung cancer. Cancer Discov. 2011;1:44-53.

21. Kris MG, Johnson BE, Kwiatkowski DJ, lafrate AJ, Wistuba II, Aronson SL, et al. Identification of driver mutations in tumor specimens from 1,000 patients with lung adenocarcinoma: The NCl's Lung Cancer Mutation Consortium (LCMC). J Clin Oncol. 2011;29(s):CRA7506. 